\title{
A Brief Overview: The Persistence and Pandemic of Virulent Newcastle Disease in Birds as Well as Role in Pakistan
}

\author{
${ }^{1 *}$ Muqadas Baksh, ${ }^{1}$ Zafar Ali, ${ }^{2}$ Ghafran Ali \\ ${ }^{1}$ Research Center for Modeling and Simulation (RCMS), National University of Science and Technology \\ (NUST), Islamabad - Pakistan. \\ ${ }^{2}$ Al-Aleem Center for Advance Studies and Research, Institute of Biotechnology, Gulab Devi Educational \\ Complex, Lahore - Pakistan. \\ *Corresponding Author: muqadas.baksh@gmail.com
}

\begin{abstract}
The poultry industry is affected by many epidemics and Newcastle Disease (ND) is a constant threat, known as a devastating disease for poultry farmers around the world. According to the average death time of chicken embryos, virus strains can be classified as lentogenic, mesogenic, or velogenic. The current research will clarify the vulnerable host range as well as the epidemiology and geographic distribution of ND in Pakistan. The introduction of the virus into poultry can have serious economic consequences, including the loss of production of sick and dying poultry, the cost of control measures (such as population reduction and disinfection measures), and possible trade restrictions in the event of an outbreak. The virus is transmitted by direct contact with sick poultry or carriers. Infected birds can also spread the virus in their feces. It can also be spread through respiratory secretions, contaminated feed, equipment, water, or feces. We will also discuss vaccines that which vaccines are available for NDV in Pakistan and vaccines can fight against this disease or not? In this study, a qualitative risk analysis was carried out to assess Pakistan's vulnerability to the introduction of virulent NDV strains.
\end{abstract}

Keywords: Newcastle Disease, Poultry, Pakistan, vaccine, Economic affects 


\section{Introduction}

The Newcastle disease was reported in Newcastle, England in 1926 and therefore it is called Newcastle disease, and in India, it was reported in 1927 from Ranikhet and is called Ranikhet. Hence, Newcastle disease is also commonly known as Ranikhet disease in India and Pakistan. Disease virus (NDV) is a virus with global distribution. Since then, three major panzootic of ND have been recorded and currently exist on every continent except Antarctica. Newcastle disease (ND) is a disease that is economically important and highly contagious to wild and captive birds. Newcastle disease (ND) occurs in poultry and may have a devastating economic impact on global poultry production. Newcastle disease virus (NDV) was formerly synonymous with avian paramyxovirus type 1 (APMV-1). However, the taxonomic change is now called avulavirus (Amarasinghe et al., 2017). According to the average death time of chicken embryos, NDV can be divided into three different pathogenic types: lentogenic (40-60 h), mesogenic (60-90 h), and velogenic (90-150 h) (Hanson \& Brandly, 1955).

Newcastle disease, also known as avian avulavirus, is caused by an antisense, single-stranded RNA virus that can infect over 250 different bird species (Hanson \& Brandly, 1955). This virus is found all over the world and has been associated with domestic chicken epizootic outbreaks on six of the seven continents (Miller, Decanini, \& Afonso, 2010). Six genes and their related structural proteins are included in the genome: (1) a nucleocapsid protein (NP), (2) a phosphoprotein (P), (3) a matrix protein (M), (4) a fusion protein (F), (5) a hemagglutinin-neuraminidase protein (HN), and (6) an RNA polymerase (L) (Chambers, Millar, Bingham, \& Emmerson, 1986; Seal, King, \& Sellers, 2000).

Amino acids that increase cell virus and cell-cell interaction and fusion have been identified as the molecular basis for virulence variation in the cleavage region of the fusion protein (Huang et al., 2004). The hemagglutinin-neuraminidase protein also facilitates NDV tissue tropism and virulence by boosting F protein fusion activity, facilitating the penetration of host cell, and inhibiting self-agglutination, sialic acid is removed from progeny virus (Huang et al., 2004).

NDV can be categorized into distinct groups and further differentiated into up to 19 genotypes based on genetic variation, with two types of AAvV-1s (I and II) as well as several genotypes and sub-genotypes. Phylogenetic analysis of incomplete or full Fusion (F) gene nucleotide sequences has led to several classification systems being proposed (Aldous, Mynn, Banks, \& Alexander, 2003).

For Class I viruses, there is only one genotype, while for Class II viruses, there are up to 18 genotypes. This system is based on full F gene analysis (I to XVIII) (Diel et al., 2012). 
Pakistan has a varied range of genotypic and sub-genotypic records which include VI, VIId, VIIf, VII, and XIIIb. Prior studies report that highly infectious sub-genotype VIIi AAvV-1s has been isolated and characterized from Pakistani pheasants, peacocks, parakeets, parrots, and pigeons, along with cases of genotype VI (Shabbir, Zohari, et al., 2013). However, the exact time that genotype VII first appeared in Pakistan is still unknown. When it comes to NDV outbreaks, VII is the most common genotype found in Europe, Asia (Tan et al., 2008), and South Africa (Abolnik et al., 2004). The sub-genotype identified from the Far East and Israel has been termed as VIId while those identified from Iran and the Indian sub-continent are termed as VIIb.

\section{Epidemiology (Pakistan)}

Newcastle Disease was named after Newcastle, England, where it was first discovered in 1926, and Ranikhet, India, where it was discovered in 1927, hence the name Ranikhet. Furthermore, first discovered in Pakistan in 1963, ND outbreaks have been documented regularly in both commercial and backyard poultry flocks alike. (Figure 1: Outbreaks in Pakistan) Depicts outbreaks across Pakistan.

\subsection{Commercial broiler and layer flock NDV outbreak incidence (1986 to 1990)}

Live bird transport and mechanical transmission of infection by standard business practices are the two most important factors in the spread of ND within a region (Dawson, 1973). In the course of an epidemic in Great Britain, evidence has also been presented for the airborne spread of NDV. (Hugh-Jones, Allan, Dark, \& Harper, 1973). In Pakistan, both vaccinated and unvaccinated flocks have been found to be infected with NDV. Commercial broiler and layer flock NDV outbreak incidence is up to $38 \%$ annually (Anjum, 1990) while commercial broiler chicken mortality within flocks can reach 50\% (Siddique, Sabri, \& Khan, 1986).

Consequently, it appears to be found that Layer and broiler production capabilities are impeded by NDV. Meanwhile, a prospective study was done in the Islamabad Capital Territory (ICT) to estimate the risk of NDV outbreaks in broiler flocks. Between July 1989 and April 1990, data on 131 flocks' demography, management practices, prophylactic procedures, and concurrent diseases were collected. During June 1989, extensive field visits to four Islamabad Capital Territory townships resulted in the enrollment of one hundred and twenty farmers who were willing to participate in the project. Farmers were informed of the study's goals during initial site visits, and they were instructed to keep all records required as a part of the study and it was discovered that $16.0 \%$ of the total population was affected by NDV attacks (Akhtar \& Zahid, 1995). 


\subsection{NDV and avian influenza serotypes (1995 to 2009)}

The samples were passaged in chicken embryonated eggs that were between 9 and 10 days old. Only fortyseven of the eighty samples tested positive for NDV using ND virus polyclonal serum used in the hemagglutination inhibition assay. Only $55 \%$ of people tested positive for ND, with $83 \%$ having solely NDV infection and the remaining $17 \%$ having a mixed infection with one of the avian influenza serotypes (T. A. Khan, Rehmani, Ayaz, Lone, \& Khan, 2012).

\subsection{Outbreaks in different regions of Pakistan (2005 to 2008)}

In 2005 field survey in Sheikhupura district, identified the prevalence of infections in local and Fayoumi breeds of poultry (Mustafa and Ali, 2005). Fayoumi breeds had a higher disease prevalence (57\%) than Desi breeds (43 percent) while 40.33 percent of the population was infected with some form of other diseases, according to the data. Several infectious agents such as NDV, AI, and E. coli, and Salmonella were discovered to be liable for respiratory epidemics in layer flocks in the Sammundri Area, according to a study done in 2008. Inadequate vaccination management along with, not healthy feed and housing due to unsatisfactory farm management combined with the failure to comply with biosecurity measures along with the movement of wild and migratory birds being the main causes of infection outbreaks in rural poultry (Khawaja, Naeem, Ahmed, \& Ahmad, 2005).

Between June 2007 and May 2008, the Khushab district Livestock Department conducted a study based on postmortem and laboratory diagnosis of poultry disease to discover common distinct poultry diseases among broilers and layers. A survey of 360 henhouses served as the basis for the prevalence analysis. Diseases in poultry were diagnosed using a combination of historical, medical symptoms, post-mortem assessment, cultural characteristics, and biochemical depictions. Broilers had the highest overall incidence (avg. 7.85 percent) of Newcastle disease. Low infectious incidences were observed from April to June when the broilers appeared to be safer. Furthermore, when dealing with several layers, the prevalence of ND was highest (avg. 7.92 percent). In conclusion, a wide range of diseases affecting chickens of all kinds, therefore proper vaccine management along with brooding plans, as well as Preventive biosecurity measures and practices should be observed (Abbas et al., 2015).

From October 2007 to February 2008, researchers in the Karachi area collected 50 tissue samples, most of the tracheal, from poultry farms with high mortality. Liquid nitrogen containers were used to transport the properly labeled samples to the lab for further processing. Results show that $40 \%$ of the samples tested 
positive for NDV, while the remaining $4 \%$ tested negative. Newcastle disease decimated Pakistan's south from October 2007 to February 2008, particularly in Multan (Ahmed et al., 2009).

\subsection{More than 1 outbreak (2008 to 2010)}

Pakistan's most common poultry disease is Newcastle disease, which is found in both urban and rural areas. Forty-five percent of unvaccinated rural poultry in the Faisalabad district tested positive for ND seroprevalence (Arshad, 2000). According to (Azhar, 2006), antibodies against the ND virus were found in 41.33 percent of unvaccinated rural chickens. Unvaccinated birds are more susceptible to the disease, which has a 100 percent morbidity and mortality rate in its most severe forms (Alexander, 1997).

In 2008, disease outbreaks were found in four villages out of the eight that had been chosen. In four villages, a total of 457 families raised rural birds, and of those, 301 (65.7 percent) had poultry birds that were infected with this disease, which resulted in an annual mortality rate of 54 percent.

Researchers in Faisalabad evaluated the epidemiology of ND in rural poultry from January to June 2009. A virus was isolated and identified from forty infected samples taken from two outbreaks. Both outbreaks took place in different towns. The outbreak hotspots were immediately investigated. On January 5, 2009, when the weather was foul and foggy, one household in a rural area began keeping unvaccinated birds in horrendous conditions in a darkened and filthy congested shack. The overall mortality rate was $65 \%$, with $76 \%$ of the chicks, $55 \%$ of the adult males, and $65.5 \%$ of the adult females dying. On the 15th of May, there was a second epidemic, this time in a different community. Within ten days, 68 percent of the village's families were seriously affected, with the infection killing 49 percent of mature male birds, 52 percent of mature female birds, and 80 percent of chicks. Chick mortality in the first outbreak was 21.7 percent, mature male mortality was 21.4 percent, and mature female mortality was 56.8 percent, whereas chick mortality in the second outbreak was 78.6 percent, mature male mortality was 51.8 percent, and mature female mortality was 56.8 percent. In each of the outbreaks, $67 \%$ of the flocks were afflicted (M. Y. Khan, Arshad, Mahmood, \& Hussain, 2011).

In Rawalpindi, a city in Pakistan's northern Pothohar plateau and a major commercial center for poultry production, an outbreak of NDV occurred in April 2010 (Munir, Zohari, Abbas, \& Berg, 2012). 


\subsection{NDV; V(i) (2009 to 2012)}

At various points between 2009 and mid-2012, several cases of Newcastle disease in Pakistan and neighboring countries were recounted to the World Organization for Animal Health (OIE-WAHID interface, available at: http://www.oie.int/wahis/public/php). Nearly all the outbreaks were found in Iran and India, which is located on the other side of Pakistani territory. Despite this, a handful of the accounts were only from a few specific places, and only the development of novel NDV (V(i)) was from Pakistan (Munir, Cortey, et al., 2012).

\subsection{NDV Velogenic and the molecular characterization of NDV (2011 to 2012)}

An ND outbreak caused by the AAvV 1 strain emerged in Pakistan's Jallo Wildlife Park in Lahore in 2012. Approximately 190 peacocks died within a week, with a 100\% fatality rate and with a $50 \%$ reduction of those birds who were vulnerable. Virus isolation and serological tests such as the HI Test, ELISA, and realtime PCR were used to establish the presence of velogenic NDV (Munir, Shabbir, et al., 2012). In just three weeks, and ND outbreak in Pakistan's Sindh province killed 167 wild peacocks in seven southern districts (Munir, Shabbir, et al., 2012).

During the NDV pandemic from 2010 to 2011, 130 specimens from the trachea, lung, spleen, cloaca, and blood were taken from 5 suspected cases of NDV infestations, including commercial broiler $(n=3)$ and layer farms $(n=2)$. Broiler flocks had a fatality rate of 10 to 15 percent per day, while layer flocks had a fatality rate of 5 to 8 percent per day. Signs of neurotropic NDV like Torticollis, head tremor, dullness, depression, and difficult respiration were all observed across all the birds. Vaccination of the infected flocks was carried out by using a commercial lentogenic strain (LaSota) in drinking water. The lentogenic strain (LaSota), which was a commercially available vaccine and mixed with the drinking water of the flock. Moreover, to combat the NDV outbreak in central Punjab, Pakistan critical information regarding the growth and spread of NDV is provided in this paper.

Furthermore, NDV genotype VII remains prevalent in Asian domestic poultry, which doesn't differ from the isolates originally depicted in the late 1990s. Due to these findings, authorities are urged to closely monitor poultry and to immediately notify the OIE for appropriate identification and monitoring with potential vaccination approaches. (Shabbir, Abbas, et al., 2013). 
In the years 2010-12, there were numerous disease outbreaks in Pakistan. Pakistan's Punjab province, where velogenic NDV strains have been discovered, has seen the majority of cases of the disease reported worldwide. Signs and symptoms of the disease were still observed in both inoculated and non-inoculated flocks of commercial, wild, and backyard poultry birds. That's the first time the molecular make-up has been characterized for the most common NDV strains found all over Pakistan. During a disease outbreak in Pakistan from February to July of 2012, blood samples were collected to determine the genetic makeup of the circulating NDV strains.

A sum of 113 flocks was assessed throughout Pakistan's four provinces (Balochistan, Khyber Pakhtunkhwa, Sindh, and Punjab), with rural $(n=42)$ and commercials $(n=60)$ birds such as pigeons, turkeys, and peacocks $(n=11)$ not being thoroughly explored. A brief medical history was taken, including the infected age along with any clinical indications of symptoms including the number of infectious deaths. Clinical screening is performed for 23 samples for F and M-based genes discovery via real-time PCR which was then found to be positive. In all provinces, real-time PCR detected samples results were shown from rural poultry and commercial. Only captive wild birds from Punjab province, however, were found to be infected. In most cases, the $\mathrm{Ct}$ values for positive samples were in the range of 25 to 30 . When tested at ambient temperature, the FTA Indicators were found to be an effective sampling system (Shabbir, Abbas, et al., 2013).

\subsection{Avian avulavirus 1 sub-genotype VIIi (2011 to 2016)}

Between 2011 and 2016, Panzootic Avian avulavirus 1 (AAvV-1) sub-genotype VIIi virus was extracted from commercialized chickens along with other non-poultry birds in Pakistan, to confirm the epidemiological link between the Newcastle epidemic and high pathogenicity in the poultry industry. According to the findings of this study, Newcastle disease is endemic to multiple hosts and environments in Pakistan (Wajid et al., 2017).

\section{Transmission (Pakistan)}

A virus shed in infected birds' droppings and respiratory secretions spread NDV for varying periods (Alexander, 1988; Hunter \& Atkinson, 2007; Strausbaugh \& Berkelman, 2003). The virus was detected in all sections of the carcass and can live for several months on the skin and bone marrow of chickens if remained refrigerated (Strausbaugh \& Berkelman, 2003). 
Infectious viruses have been shown to survive in poultry houses contaminated by infected birds for 7 days in summer, 14 days in spring, and 30 days in winter (Kinde, Utterback, Takeshita, \& McFarland, 2004). In gallinaceous birds such as chicken and turkeys, pheasants, and partridges, grouse, the infected virus can be released for up to one to two weeks after infection. Although, psittacine birds such as parakeets, parrots, and macaws have shown the ability to propagate transmissible viruses mainly through respiratory secretions and feces, from months to several years after infection. As maternal antibody levels decrease, the mortality rate of chicks with severe NDV infection will increase. The virus can spread from one location to another by using contaminated feeding equipment, bags, or water, as well as human movement, egg crates or cages, visitors, or owners. Migration and waterfowl can also spread the disease.

\section{Newcastle disease virus in other avian species}

The fatality rate and clinical symptoms differed greatly even among flocks of the same species, including large flocks of inoculated chicken, and non-inoculated pet birds. Numerous bird species and ages have been identified as being virus-infected. Symptoms of ND were evident in infected birds, including respiratory, neurological, and/or enteric signs (Table 1: Clinical Signs of different species due to NDV). Both vaccinated and unvaccinated chicken flocks showed symptoms of ND.

\subsection{Partridges and pheasants}

NDV is extremely contagious, particularly in partridges and pheasants. Clinical symptoms are similar to those seen in chickens and they can vary with an early onset accompanied by rapid death to severe neurological symptoms and subclinical infection. In addition, the lesions resemble those found in other poultry.

In Lahore in 2012, there was a suspected AAvV-1 epidemic that killed 67 pheasants within 2 weeks after the beginning of clinical symptoms. The apparent case fatality rate was 80 percent, with a loss of 60 percent of the vulnerable birds. (Shabbir et al., 2012).

\subsection{Waterfowl: Ducks}

It is well known that domesticated waterfowl and other poultry and non-poultry species play an important role in spreading avian viruses among wild waterfowl. During the winter months of late November, many migrating waterfowl arrive in Pakistan from northern Russia and scatter across the country. Over the "Hindu Kush", "the karakorum", and "Suleiman Ranges of the Indus River". Birds migrate from Siberia to Pakistan alongside the Indus Flyway and International Migratory Bird(IMBR) no. 4 (Wajid, Dundon, Hussain, \& 
Babar, 2018). To better understand how these migratory wild bird species influence disease transmissions in Pakistan, more research and surveillance are needed.

After contracting the virus, ducks may aid in its spread. Only one study has documented neurological symptoms in ducklings experimentally infected with a mesogenic strain that had previously caused an outbreak among ducks. When infected with a highly pathogenic NDV, ducks generally show no symptoms of ND (P. Miller \& G. Koch, 2013).

\subsection{Peacock}

NDV genotype VII and subgenotype 'e' were found to be present in a peacock isolate from the United States. The NDV virus is widely distributed in Pakistan, and outbreaks in wild poultry flocks, rural poultry flocks, and commercial poultry flocks are regularly reported.

Between 2012 and 2013, researchers discovered the ND endemic in Pakistan's Tharparker desert's peacocks, which are thought to number in the tens of thousands. Since the beginning of June, the deadly New Castle disease has claimed the lives of over fifty Hyderabadi peacocks. A vitamin and mineral deficiency is to blame for the disease, which influences the birds' nervous systems. This prompts the peacocks to become dizzy and die by suffocation. Acute water scarcity, scorching desert heat, and low millet and maize yields are all contributing factors in the nutritional crisis (Table 2: Reasons for Peacock's ). In 2012, a similar outbreak claimed the lives of 300 peacocks, but the wildlife department disputed the exact number of birds that perished.

Asian Scientist (Aug. 13, 2012) - Every year, wild peacocks in Pakistan's Tharparkar Desert celebrate the arrival of the monsoon season. A deadly disease spread across the desert-like wildfire, however, and the celebrations were cut short that year. At least 167 people have died according to unconfirmed reports because of Newcastle Disease, according to the Karachi-based Poultry Research Institute. Tharparkar, Mirpurkhas, Tando Allahyar, Badin, Sanghar, Hyderabad, and Shaheed Benazirabad are the seven districts in Sindh's southern region that have been affected by the disease. Gullan Jattar, Bapuhar, and Hoti Jattar are among the worst-hit villages out of the 2,300 total in these districts. Peacocks are reportedly found in over 1,400 villages in Thar, or about $60 \%$ of the district ("Newcastle Disease Linked To Peacock Deaths In Pakistan,").

According to the wildlife department's estimates of 80,000 populations have been recipients of medicine in their water. However, no system exists to identify and track the birds who have been given the vaccine. ND 
endemic peacocks are among the 40,000 species found in Pakistan's Sindh province's Tharparker desert. Tharparker has not conducted an epidemiological study on the NDV epidemic in peacocks. As a result, the goal of this investigation was to identify the epidemiological factors that contributed to the NDV outbreak. As summer approaches and temperatures rise in the Thar Desert region, there has been an increase in reports of death among the region's indigenous birds, peacocks, and their offspring, in Sindh's Tharparkar district. While the exact cause of the birds' deaths is unknown, the activist believes it was due to the region's hot weather and drought-like conditions, as well as a possible outbreak of the highly contagious Newcastle disease ("NDV in peacock,").

\section{Vaccines}

In the poultry industry, vaccines are used to prevent and manage infectious diseases, hence a precise vaccination schedule must be followed to minimize or eliminate the occurrence of clinical disease at the farm level. Vaccination programs for domestic poultry are implemented over the world and be effective in preventing clinical disease. Variations in vaccination regimens, vaccines, and endemic strains are used throughout the world according to local poultry-production habits and cold-chain availability, but the disease still presents a threat to the poultry sector. A healthy flock of chickens can contract the disease from sick neighbors' pigeons, parakeets, and backyard poultry, as well as from crows, kites, and sparrows, doves, and other bird species. LaSota strain of ND vaccine has been widely used for decades to combat NDV epidemics, and it may be administered through drinking water with no human labor and low impact on hen flocks. Many nations, including Pakistan, use live viral vaccinations and dead oil-based vaccines to combat chicken sickness.

But despite the widely used vaccination campaigns, epidemics continue to take place, causing huge economic losses across the country. Historical and Clinical indicators, physical examination results, and laboratory confirmation are used to diagnose the condition.

Prior Studies (Ali et al., 2014) have established that a strict vaccination schedule combined with an inactivated ND vaccine is more efficient than a live attenuated version in decreasing/eliminating ND virus shedding and multiplication. Furthermore, it is more effective to utilize homologous strains for immunization, as it provided 90 to 100 percent protection and less harmful effects on maternal immunity than live vaccinations, which only provided 50 and 60 percent protection at 28 and 42 days of life, respectively. 


\subsection{Vaccines solved the problem?}

ND control should consist of strict biosecurity measures to prevent toxic NDV from contacting poultry and to properly vaccinate effective vaccines. Since the 1950s, live vaccines and inactivated vaccines have been utilized commonly. Usually, infected poultry leads to morbidity and death due to incomplete or inadequate vaccination. Another reason for the poor effectiveness of the vaccine in the presence of antibodies (including the mother) in the poultry, which can deactivate the vaccine and reduce the efficiency of the vaccine for the ND. Clinical diseases affecting the nervous system, gastrointestinal tract, reproductive system, and respiratory system are most common in unvaccinated, unvaccinated, or unvaccinated chickens (P. J. Miller \& G. Koch, 2013).

The vaccine of the Newcastle disease has three main goals. i) reduce clinical disease, ii) reduce the discharge of toxic virus, iii) increase capability to attack virus (Kapczynski, Afonso, \& Miller, 2013). veterinarians do not have the latest technology to consider the effectiveness of vaccination for achieving the second and third goals and just $1^{\text {st }}$ goal is considered for the current control strategy.

Importantly, vaccinated poultry is considered to be the host of the virulent strain of NDV, because it can infect the virulent strain after vaccination and spread the infectious virus without clinical disease. In recent years, it has been observed that LaSota strains cannot protect against lethal field strains that are particularly resistant to NDV in broilers. The reasons for immunosuppression include exposure of poultry to bacteria, viruses, or other toxic substances, rather than poor management, including the negligence of poultry farmers.

\subsection{Occurrence of Virulent NDV in Inoculated Chickens}

A prior study (Rehmani et al., 2015) concluded that $80 \%$ of farms vaccinated chickens with LaSota live vaccine on the 14th day through spray or eye drops in the hatchery and by water on the 14th day. $20 \%$ of farms received an additional dose of inactivated vaccine on day 24. On-site HAI and matrix PCR tests showed that regardless of the age of the chickens (broiler and layer), 88 of the 101 farms evaluated tested positive for NDV, and 20 of the 88 farms confirmed the virulent virus. It is assumed that these groups are not infected with vNDV, but since Pakistan is an endemic area of NDV, the origin of the outbreak is still unclear. Poultry mortality is between $6 \%$ and $50 \%$, and records are showing that although some farms have been vaccinated, the morbidity rate can still reach $60 \%$. In the most severe cases, the onset occurs between the 21 st and 25th days of life and the death occurs within 10-15 days. No bird flu was detected. 


\section{Economic effects}

The Poultry sub-sector is an important backbone for an agricultural country like Pakistan where shortfalls in protein deficiency are fabricated through poultry production which provides a cheap source of protein in the form of meat and eggs. Every household in the countryside and every 5th household in the city is connected in some way with their poultry production activities. With a yearly growth of 7 to $8 \%$ Poultry stipulates for about $26.8 \%$ of the country's overall meat manufactured. Pakistan's poultry production is rising. One of the reasons for the decline in poultry production is an infectious disease. The Pakistan Economic Survey (2011-2012) reported that by 2012, the poultry industry will generate enough wages for the direct and indirect employment of approximately 1.5 million people.

Poultry accounts for $6.40 \%$ contribution in agriculture along with $11.50 \%$ in animal husbandry. Generally speaking, rural meat and poultry production accounted for $25.8 \%$. The poultry industry is growing rapidly at an annual rate of about 8-10\%, showing unique potential. Prior research shows that about Rs. 200 billion have been invested in Pakistan's poultry sector. ND is one of the major bird virus diseases that cause huge economic losses. ND is a contagious and reportable disease. It is likely to spread very quickly and widely, and have a socio-economic impact on the animal's global trade. ND and other OIE-listed diseases, such as avian influenza (AI) that have been reported in Pakistan have harshly affected the supply and demand of the poultry sector.

\section{Diagnostics}

Various analytical devices have been introduced to describe infectious agents. These techniques are regularly used continuously. sick and dying birds' samples collected can be inoculated into embryo eggs and further differentiated. Mean death time (MDT) is a commonly used analytical method to determine the time (in hours) required to inoculate a sample into a hatching egg and kill a chicken embryo. The intracerebral pathogenicity index (ICPI) and intravenous pathogenicity index (IVPI) include weighted scores of clinical symptoms in newborn chicks after intracerebral or intravenous injection, respectively. ICPI is the preferred test for NDV, while IVPI is only used sporadically and has never been used for formal purposes.

Virus isolation is the golden benchmark for diagnosing the NDV which is analyzed by hemagglutination and inhibition of hemagglutination. From a serological point of view, hemagglutinin inhibition (HI) assay 
or enzyme-linked immunosorbent assay (ELISA) can be employed to assess the occurrence of NDVspecific antibodies in the host. Both HI and ELISA diagnoses can be used to detect antigens or antibodies. Compared with virulent NDV isolates, serological diagnosis is not very effective in determining that NDV is the cause of a specific outbreak due to widespread vaccination, because it cannot distinguish the antibodies produced by exposure to the vaccine strain. Many commercial operations routinely use ELISA to closely monitor the serology of slaughtered flocks. Based on many years of data, the normal range of NDV antibody titer has been determined. If it exceeds this value, further testing and in-depth evaluation are required. Such methods facilitate distinguishing irregular situations swiftly and muster responses when incidents occur.

\section{Prevention and Control}

To control and prevent ND, vaccines can be used. In rural regions, the lack of knowledge about the Newcastle disease vaccine is the main factor that is playing role in the development of Newcastle disease. To prevent chicks from being infected with ND, the flocks must be vaccinated against Newcastle disease. The local strain of Newcastle disease vaccine distributed in various regions can be used for Newcastle disease vaccination. Improper vaccination can lead to the development of ND. Adequate vaccination can protect birds from diseases, but it cannot prove a barrier in the replication and shedding of the virus and may lead to infection. ND is the main problem that is susceptible to ND in rural livestock in Pakistan, especially Aseel (Desi) poultry. The recent NDV strains which have no or very poor immunity to local birds will cause the vaccination of rural poultry to fail. In rural poultry flocks, regular vaccination of NDV can help prevent ND. The common local ND vaccine helps protect chickens from local strains which is available in the market. Vaccination is should be used according to the age of the poultry and given the manufacturer's recommendations and instructions. The route of vaccination is eye drops (eyes), intranasal (spray), subcutaneous injections, and drinking water. Use of the ND vaccine in drinking water, User should stop use medications and disinfectants in drinking water before 24 hours of drinking, and use it again 24 hours after vaccination. Vaccination requires the use of non-chlorinated water. Failure of vaccines can lead to the development of ND. Despite vital and regular vaccination, ND still causes serious damage to poultry flocks and outbreaks have also enveloped the vaccinated flocks. Reasons for vaccination disappointment include incorrect vaccination methods, use of vaccination amid simultaneous incubation periods of the new coronavirus, stress from thrilling weather conditions in winter and summer, and after vaccination, instant poultry transportation. Low vaccine quality is also one of the reasons for vaccination failure. 
Poor quality vaccines are due to insignificant making standards, low numbers of antigens, expired vaccine's utilization, improper use in poultry, unavailability of adequate storage, improper supervision of vaccines during transportation, direct sunshine, and unmaintained resource cold chains. Failure and Disappointment of Vaccine can also be because of control caused by mycotoxins and/or drugs, pressure on birds, coinfection factors, failure of "host defense mechanisms", the existence of high maternal antibody titers. Vaccine failure can be prevented by monitoring the quality of the vaccine and the host immune system can prevent ND in poultry flocks. Since dead birds are the source of ND transmission, they will form death pits in the walls of your farm. All dead birds should be buried in pits to properly discarding and prevent the spread of ND (Ashraf \& Shah, 2014).

\section{Commercial poultry}

Newcastle disease is an extremely contagious disease of many birds and is the cause of fatal disease outbreaks, especially in Pakistan's commercial poultry populations, in Pakistan's poultry industry causing giant economic losses. Despite extensive vaccination programs for poultry, the disease still appears to be endemic in commercial broiler and layer farms. The research was directed to determine the epidemic velogenic NDV strain that caused the disease outbreak in a commercial poultry farm of Punjab in Pakistan.

This was the first report to show that among all viral, bacterial, and parasitic diseases in Pakistan, the presence of ND is considered the main source of infection for commercial poultry (Rehman, 2013). The prevalence of ND in broilers and laying hens remains high all over the year but has reached a peak due to seasonal pressures (January-February and June-July).

Between September of 2011 and January of 2012, 45 million chickens were died due to ND in Punjab, Pakistan alone, faced the loss of US\$65 million in the poultry industry. ("Newcastle disease hits Pakistan poultry http://www thepoultrysite.com/poultrynews/25595/newcastle-disease-hits-pakistan-poultry.,"). However, under the same environmental conditions, breeder flocks remain healthy, which emphasizes the importance of implementing good management practices and biosecurity measures in breeder farms (Rehman, 2013). The role of exotic and wild birds in the epizootics of ND is not aware of, but it is speculated that wild birds may play an important role in spreading the virus from affected poultry farms to unaffected family farms. The last reports of the commercial, backyard, and wild poultry in different regions of Pakistan point toward the occurrence of multiple genotypes (Munir, Abbas, Khan, Zohari, \& Berg, 2012; Munir, Cortey, et al., 2012; Munir, Shabbir, et al., 2012). 
In the early 1960s, Commercial poultry farming in Pakistan started and has developed rapidly over the decades. The early development of the industry benefited from the government's support policies and the persistence of the poultry farming community. The government regards the poultry production chain as an important part of the food processing industry and provides special incentives for the industry. Therefore, the poultry industry got freedom from sales tax and income tax, and exemption from import duties for many years (Sadiq, 2004). According to (NAZIR, 2013) Pakistan has not succeeded in producing SPF-free chickens, and feed prices are high (Tauqir \& Nawaz, 2001).

\section{Disease awareness}

Approximately $12 \%$ of households are nescience about ND and poultry care, while $88 \%$ of households have little knowledge of disease and management. In $8 \%$ of households, men keep birds, while in $92 \%$ of households, women are responsible for maintenance. Compared with households that regularly vaccinate poultry with ND, the mortality rate of households not vaccinated with ND is relatively higher. During the breeding season, farmers who kept poultry in dark, crowded, and dirty houses at night had the highest mortality rate. Farmers who keep birds in proper shelters have lower mortality rates. Families with little knowledge of the disease took various measures to protect their flocks from the disease, so their attempts were somewhat successful, while birds ignorant to the disease were the victim of ND. Lack of immunizations, unavailability of cold chain facilities, year-round birds, inadequate living in crowded and dirty homes under severe weather, insufficient feeding, unethical handling of dead birds at home, and other basic treatments. Ignorance of agricultural facilities is one thing. A highly fatal outbreak and alarming death rate (M. Y. Khan et al., 2011).

\subsection{Role of paddler's birds}

Paddlers are sold, and their cages and birds are an everlasting source of infection. It is reported that compared with families that raise chicks with existing birds compare to families rearing birds from broody hens in the houses (M. Y. Khan et al., 2011). 


\subsection{Weather effects}

Extreme weather in summer and winter can weaken the immune status of birds to survive under adverse conditions such as lack of shelter, insufficient food and water supplies (M. Y. Khan et al., 2011). According to reports, it occurred under severe weather conditions during extremely hot and cold seasons. (Mishra \& Spradbrow, 1991) found that compared with other seasons, ND occurs more frequently in summer. Many authors have reported the influence of seasonality on the incidence and severity of ND. (Nguyen, 1991) reported that ND outbreaks were more widespread in the winter in Vietnam (Musiime, 1991)also determined that ND infections were more frequent during cold and dry periods. In this study, the emergence of rural birds arose all over the year. However, the frequency is higher in hot and cold months.

\subsection{Gender difference}

The mortality rate in young birds from ND was much higher than that in older birds. ((Ezeokoli, Umoh, Adesiyun, \& Abdu, 1984) The results of these studies are that poultry at approximately 16-24 weeks of age in a backyard management system had the hazardous NDV infection. Some studies have shown that the sex of birds can influence the morbidity and mortality of the ND virus. (Kutubuddin, 1973) A pathological study was performed on a poultry farm at the Bangladesh Agricultural University. He found that male birds were more susceptible to NDV than female birds. However, according to a statistic study, there was no statistically significant difference in ND mortality in unvaccinated or vaccinated males and females in this study, but higher mortality in chicks (M. Y. Khan et al., 2011).

\section{Risk analysis}

Introducing highly virulent avian viruses have increased due to Globalization, international trade, and migratory bird species with suspected NDV infection. Many methods require attention, including introduction through live poultry or poultry product shipping points, and the continued presence of viruses on contaminated or contaminated poultry food, water, or supplies. Closed houses, indiscriminate vaccination, inadequate biosecurity management, unhygienic practices, the introduction of wild birds into farms, and lack of diagnostic facilities are the main reasons for the spread of NDV in Pakistan. Pakistan should develop a comprehensive national policy for the disease-free poultry sector. 


\subsection{Poultry premises risk}

Pakistan's poultry industry is characterized by sizes of farms ranging from more than 1,000 to less than 10,000 chickens. In general, the average flocking force of broilers and laying hens is about 2,000 to 3,000, which is called rural farming. These types of farms are characterized by household labor and often with a lack of modern technology. In recent years, poultry companies have developed rapidly in Pakistan. However, various infectious diseases pose a serious threat to the survival of poultry breeding, especially the small-scale breeding industry, and cause serious economic losses to the country. The main poultry disease includes "Newcastle disease (ND)", "Escherichia coli infection", "Infectious coryza", "Infectious bronchitis (IB)", "Coccidiosis", "Enteritis", "Fowlpox", "Salmonellosis", "Hydropericardium syndrome (HPS)", and "Avian Influenza (AI)". A very important environmental factor is the season that can greatly influence the incidence of poultry diseases.

Previous surveys in the NDs have reported the occurrence of poultry diseases and their relationship to age and weather. Most backyard poultry farming contains a small of 10 to 15 head units in Pakistan. Because they are primarily grazing, they generally lack the biological safety and good breeding practices to prevent the spread of NDV. Also, traditions, cockfights, and hobbies (dove racing and performance), such as using exotic pet birds as status symbols, can facilitate the activity of infected pet birds.

\subsection{Domestic's backyard poultry management risk}

In the era of protein shortage in Pakistan, poultry eggs and meat are valuable sources of protein. Rural poultry products have been at the forefront of consumers because of their delicious taste. Pakistan's poultry industry is the backbone of our business and rural economy. It has made a significant contribution to the country's GDP. There are about 1105.91 million poultry birds in Pakistan, of which approximately 15.244 million are rural poultry. It plays an important role in the town's economy, producing as many as 3611 million eggs and 100.41 metric tons of poultry meat. Newcastle disease is the most common poultry disease in rural Pakistan. The biggest impact of ND is the high mortality of poultry in the village, which reduces the supply of eggs and meat protein. Poultry production in rural or small farmers is the main source of protein and income for small farmers, and the outbreak of ND has led to food insecurity in these communities. The seroprevalence rate of ND in unvaccinated rural poultry from Faisalabad was 40.5 (Arshad, 2000). (Azhar, 2006) reported that $41.33 \%$ of unvaccinated rural chickens were found to be 
positive for antibodies to the ND virus. In severe forms of the disease, morbidity and mortality can reach $100 \%$, and unvaccinated birds are more susceptible to the disease (Alexander, 1997).

\subsection{Laboratory risk}

Due to the high risk of ND virus transmission in laboratories, a risk assessment should be performed to determine the level of biosafety and biosecurity required for virus diagnosis and identification. Biosafety and Biosecurity: A standard for biohazard management in veterinary laboratories and animal facilities. The facility must operate at Biosafety Level 2 or higher. Countries, where such specialized national or regional laboratories are not available, must send samples to the OIE Reference Laboratory.

Newcastle disease is a viral disease that affects the birds and left a severe impact on the economy of the poultry industry. To control the economic losses and disease, Fast and reliable detection and identification of ND is helpful. It is essential to raise awareness among field staff and use the most effective and accurate laboratory testing procedures due to NDV can cause a variety of disease symptoms. For identifying disease in the field and developing a differential diagnostic checklist, understanding NDV pathology is necessary.

It is clear from the literature that rapid detection, typing, and subtyping of NDV remains a challenge in the laboratory because of the diversity of disease manifestations and lesions as well as the genetic diversity of the strains involved. This variability may be a major limitation in the validation and application of advanced molecular techniques currently used for NDV diagnosis. It also reminds us of the significance of multidisciplinary and complete diagnostic methods that should include more traditional techniques such as histopathology, IHC, and viral isolation, as well as next-generation detection methods in the genomic era.

\section{Biosecurity}

Inadequate biosecurity contributes to the spread of ND (Okwor \& Eze, 2010). ND can be stopped through strict biological security and controlling the activity of infected birds. When building new poultry farms, it is important to maintain adequate distance between each poultry farm. A broiler farm should be built $1 \mathrm{~km}$ from the nearest broiler farm, and a broiler farm should be built $3 \mathrm{~km}$ from the nearest poultry farm. The strict biosecurity measures on poultry farms help prevent viral and bacterial diseases. In this regard, in and out movement of workers should be strictly observed, and that people who don't work in the form should not be permitted go to inside of the farm. In addition, the free movement of wild birds, pet birds, watchdogs, 
and cattle in farm areas should be limited. Visitors should be minimized in the form. When visiting poultry farms, visitors must be provided with clean clothing. Breeders, electricians, and other employees of commercial poultry farms are not allowed to raise backyard poultry in their residence, furthermore should not go to live poultry bird's markets.

Employees must wear dry, clean overalls when working in the poultry house. Poultry farms must maintain good sanitation and sewage systems to prevent the spread of disease. Confirm the spread of ND in surrounding areas and take appropriate precautions to prevent ND in poultry herds (Sharif, Ahmad, Umer, Rehman, \& Hussain, 2014).

In the event of a new crown virus outbreak, zoning should be implemented to limit the movement of farm staff, workers, etc., and must be collect dead and sick birds according to standard operating procedures determined by the epidemic situation. End the epidemic by cleaning and disinfecting premises, isolating them, controlling movement, and reducing all infected and exposed birds.

\section{Proper management}

The outbreak of ND may be due to inadequate preventive measures, unsanitary conditions, poor brooder arrangement, lack of adequate spacing between successive flocks, and insufficient knowledge about the scientific breeding of rural poultry.

Many factors may influence the spread of ND in birds, including maintaining old poultry flocks (AbdulAziz \& Arp, 1983; Cattoli, Susta, Terregino, \& Brown, 2011; Okwor \& Eze, 2010). Keeping a flock of oneyear-old birds on the farm will aid in the effective management of the chicks and help complete the disinfection of the farm during the intervals between flocks. Trucks used to transport feed and feed bags must be properly fumigated and tires must be properly disinfected before entering the poultry house. Before new flocks are housed, they must be properly fumigated with a solution of formalin and KMnO4. Drinking water by birds in ponds and puddles can also be a source of bird infection. Poor ventilation and housing conditions are the reasons for the spread of ND in poultry (Khawaja et al., 2005). 


\section{Legal movement of animal's products}

Products and by-products produced in NDV-free regions may be imported in their original form. Animal feed containing poultry products should be heated at an internal temperature of $74{ }^{\circ} \mathrm{C}$ before import from NDV-endemic areas, but raw animal feed products may be imported from NDV-free areas.

\subsection{Live bird's illegal movement and their products}

Lack of government supervision, types of products being transported, countries of origin and final destination, and illegal transport of live animals and products can spread disease. According to data from the US Fish and Wildlife Service, between 2007 and 2016, nearly 400,000 specimens of wild birds were illegally imported or improperly documented and subsequently seized by the Fish and Wildlife Service. However, it is difficult to analyze and recorded that how many birds and their products were imported illegally or improperly.

\section{Future challenges}

The evolutionary origin of the Newcastle disease virus in its various outbreaks has not yet been studied. The focus of research work has been done on the immunological properties of the virus rather than its genomic properties. In addition, the common use of vaccines makes genetic modification of pathogenic strains more favorable. Therefore, it is necessary to address these issues through the following studies for international attention. "1) Isolation and molecular characterization of velogenic strains of NDV; 2) complete genome sequence analysis of different NDV isolates for further studies of epidemiology, vaccinology, and evolutionary origin; 3) existing real-time PCR assays should be validated and measures should be devised for prevention and control of epidemics in future" (Ashraf \& Shah, 2014). 


\section{Conclusion}

The highly virulent Newcastle disease virus represented by a variety of poultry virus strains is a highly contagious virus with almost equal mortality in poultry. In addition, the detection of virulent NDV is a mandatory report of the disease to OIE and leads to severe and often long-term restrictions on international trade. Pakistan should develop a comprehensive national policy for the disease-free poultry sector.

In developing countries, ND remains a major threat to the poultry industry, including Pakistan. Currently, ND is common in all areas of Pakistan, and the incidence has increased. However, it is clear from several global epidemics that current vaccination strategies are not fully effective under various environmental conditions, and new vaccine production concepts must be developed. There is a need to improve the efficacy of vaccines and thereby improve the immune response. Therefore, it is necessary to research innovative methods and develop safe new, and powerful adjuvants. The future ND vaccine system can rapidly evolve against newly emerging NDV strains and design a variety of vaccines. These vaccines have many advantages compared with today's existing vaccines. ND is a lethal disease and remains one of the main problems in the existing and developing poultry industry in many countries.

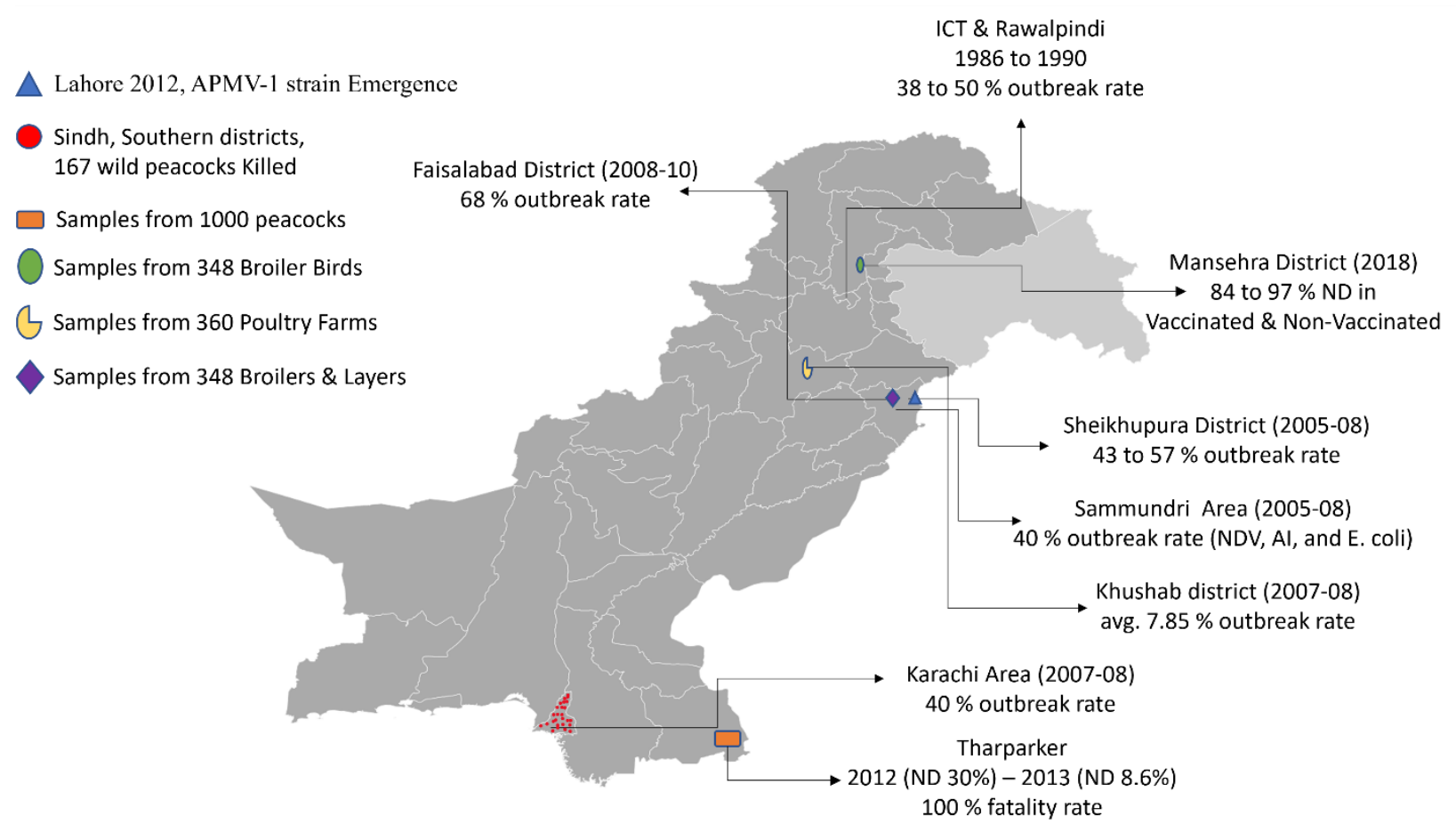

Figure 1: Outbreaks in Pakistan 


\begin{tabular}{|c|c|}
\hline Name & Clinical signs \\
\hline Partridges and pheasants & $\begin{array}{ll}\text { - } & \text { Sleepy appearance } \\
\text { - } & \text { Do not eat } \\
\text { - } & \text { Difficulties in breathing }\end{array}$ \\
\hline Chickens & $\begin{array}{l}\text { - May die without showing any signs of } \\
\text { illness } \\
\text { - Sleepy appearance } \\
\text { - } \text { Do not eat } \\
\text { - } \text { Difficulties in breathing } \\
\text { - } \text { Swelling of the head \& neck } \\
\text { production } \\
\text { - Paralysis of wings and legs Muscle } \\
\text { tremors }\end{array}$ \\
\hline $\begin{array}{l}\text { Waterfowl } \\
\text { Ducks }\end{array}$ & $\begin{array}{ll}\text { - } & \text { Respiratory failure } \\
\text { - } & \text { High level of mortality } \\
\text { - } & \text { weight loss }\end{array}$ \\
\hline
\end{tabular}

Table 1: Clinical Signs of different species due to NDV

\begin{tabular}{|c|c|c|c|c|}
\hline Name & Genotype & Clinical signs & Reasons & Outbreak \\
\hline Peacock & $\begin{array}{l}\text { VII and } \\
\text { subgenotype }\end{array}$ & $\begin{array}{l}\text { - } \text { Dizzy } \\
\text { - Whirl to their } \\
\text { death } \\
\text { - Nasal } \\
\text { discharge } \\
\text { - Swollen eyes } \\
\text { are observed }\end{array}$ & $\begin{array}{l}\text { - } \begin{array}{l}\text { Deficiency of } \\
\text { vitamins } \\
\text { minerals. }\end{array} \\
\text { - In Tharparkar, hot } \\
\text { weather } \\
\text { Acute shortage of } \\
\text { water, sweltering } \\
\text { desert heat } \\
\text { low yield of millet }\end{array}$ & 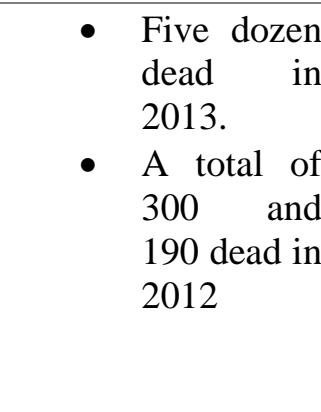 \\
\hline
\end{tabular}




\section{REFERENCES}

1. Abbas, G., Khan, S. H., Hassan, M., Mahmood, S., Naz, S., \& Gilani, S. S. (2015). Incidence of poultry diseases in different seasons in Khushab district, Pakistan. Journal of Advanced Veterinary and Animal Research, 2(2), 141-145.

2. Abdul-Aziz, T., \& Arp, L. (1983). Pathology of the trachea in turkeys exposed by aerosol to lentogenic strains of Newcastle disease virus. Avian diseases, 1002-1011.

3. Abolnik, C., Horner, R., Bisschop, S., Parker, M., Romito, M., \& Viljoen, G. (2004). A phylogenetic study of South African Newcastle disease virus strains isolated between 1990 and 2002 suggests epidemiological origins in the Far East. Archives of virology, 149(3), 603-619.

4. Ahmed, A., Khan, T. A., Kanwal, B., Raza, Y., Akram, M., Rehmani, S. F., ... Kazmi, S. U. (2009). Molecular identification of agents causing respiratory infections in chickens from the southern region of Pakistan from October 2007 to February 2008. Int J Agric Biol, 11(3), 325-328.

5. Akhtar, S., \& Zahid, S. (1995). Risk indicators for Newcastle disease outbreaks in broiler flocks in Pakistan. Preventive Veterinary Medicine, 22(1-2), 61-69.

6. Aldous, E., Mynn, J., Banks, J., \& Alexander, D. (2003). A molecular epidemiological study of avian paramyxovirus type 1 (Newcastle disease virus) isolates by phylogenetic analysis of a partial nucleotide sequence of the fusion protein gene. Avian pathology, 32(3), 237-255.

7. Alexander, D. (1988). Newcastle disease: methods of spread Newcastle disease (pp. 256-272): Springer.

8. Alexander, D. (1997). Newcastle disease and other Avian Paramyxoviridea infections. Diseases of poultry, 721-738.

9. Ali, M., Muneer, B., Hussain, Z., Rehmani, S., Yaqub, T., \& Naeem, M. (2014). Evaluation of efficacy of killed and commercially available live Newcastle disease vaccine in broiler chickens in Pakistan. The Journal of Animal \& Plant Sciences, 24, 1663-1667.

10. Amarasinghe, G. K., Bào, Y., Basler, C. F., Bavari, S., Beer, M., Bejerman, N., . . Bukreyev, A. (2017). Taxonomy of the order Mononegavirales: update 2017. Archives of virology, 162(8), 2493-2504.

11. Anjum, A. (1990). Weather and disease: 1. Prevalence of poultry diseases in and around Faisalabad and their relationship to weather. Pakistan Veterinary Journal, 10(1), 42-45.

12. Arshad, M. (2000). Epidemiology and immunoprophylaxis of Newcastle disease in poultry.

13. Ashraf, A., \& Shah, M. (2014). Newcastle disease: Present status and future challenges for developing countries. African Journal of Microbiology Research, 8(5), 411-416.

14. Azhar, M. (2006). Seroprevalence of Newcastle disease and Avian Influenza in backyard poultry. M. Sc.(Hons.) Thesis, Department of Veterinary Microbiology, Univ of Agriculture, Faisalabad, Pakistan.

15. Cantin, C., Holguera, J., Ferreira, L., Villar, E., \& Munoz-Barroso, I. (2007). Newcastle disease virus may enter cells by caveolae-mediated endocytosis. Journal of general virology, 88(2), 559569.

16. Cattoli, G., Susta, L., Terregino, C., \& Brown, C. (2011). Newcastle disease: a review of field recognition and current methods of laboratory detection. Journal of veterinary diagnostic investigation, 23(4), 637-656.

17. Chambers, P., Millar, N. S., Bingham, R. W., \& Emmerson, P. T. (1986). Molecular cloning of complementary DNA to Newcastle disease virus, and nucleotide sequence analysis of the junction between the genes encoding the haemagglutinin-neuraminidase and the large protein. Journal of general virology, 67(3), 475-486.

18. Dawson, P. (1973). Epidemiological aspects of Newcastle disease. Bulletin. 
19. Diel, D. G., da Silva, L. H., Liu, H., Wang, Z., Miller, P. J., \& Afonso, C. L. (2012). Genetic diversity of avian paramyxovirus type 1: proposal for a unified nomenclature and classification system of Newcastle disease virus genotypes. Infection, Genetics and Evolution, 12(8), 1770-1779.

20. Dimitrov, K. M., Afonso, C. L., Yu, Q., \& Miller, P. J. (2017). Newcastle disease vaccines-a solved problem or a continuous challenge? Veterinary microbiology, 206, 126-136.

21. Dortmans, J. C., Koch, G., Rottier, P. J., \& Peeters, B. P. (2011). Virulence of Newcastle disease virus: what is known so far? Veterinary research, 42(1), 122.

22. Ezeokoli, C., Umoh, J., Adesiyun, A., \& Abdu, P. (1984). Prevalence of Newcastle disease virus antibodies in local and exotic chicken under different management systems in Nigeria. Bulletin of animal health and production in Africa= Bulletin des sante et production animales en Afrique.

23. Hanson, R., \& Brandly, C. (1955). Identification of vaccine strains of Newcastle disease virus. Science, 122(3160), 156-157.

24. Huang, Z., Panda, A., Elankumaran, S., Govindarajan, D., Rockemann, D. D., \& Samal, S. K. (2004). The hemagglutinin-neuraminidase protein of Newcastle disease virus determines tropism and virulence. Journal of virology, 78(8), 4176-4184.

25. Hugh-Jones, M., Allan, W., Dark, F., \& Harper, G. (1973). The evidence for the airborne spread of Newcastle disease. Epidemiology \& Infection, 71(2), 325-339.

26. Hunter, D. B., \& Atkinson, C. (2007). Infectious diseases of wild birds. Ed. Thomas NJ Black well, Ames, lowa, USA. Pp, 137-139.

27. Kapczynski, D. R., Afonso, C. L., \& Miller, P. J. (2013). Immune responses of poultry to Newcastle disease virus. Developmental \& Comparative Immunology, 41(3), 447-453.

28. Khan, M. Y., Arshad, M., Mahmood, M. S., \& Hussain, I. (2011). Epidemiology of Newcastle Disease in Rural Poultry in Faisalabad, Pakistan. International Journal of Agriculture \& Biology, 13(4).

29. Khan, T. A., Rehmani, S. F., Ayaz, A., Lone, N. A., \& Khan, M. N. (2012). Characterization of Newcastle disease virus isolated during 1995-2009 from suburbs of Karachi-Pakistan. Pakistan Journal of Zoology, 44(2).

30. Khawaja, J., Naeem, K., Ahmed, Z., \& Ahmad, S. (2005). Surveillance of avian influenza viruses in wild birds in areas adjacent to epicenter of an outbreak in federal capital territory of Pakistan. Int. J. Poult. Sci, 4(1), 39-43.

31. Kinde, H., Utterback, W., Takeshita, K., \& McFarland, M. (2004). Survival of exotic Newcastle disease virus in commercial poultry environment following removal of infected chickens. Avian diseases, 48(3), 669-674.

32. Kutubuddin, K. (1973). Pathological investigation on the causes of mortality of chickens in the BAU poultry farm. M. Sc. Thesis, Department of Pathology, Faculty of Veterinary Science, Bangladesh Agricultural University, Mymensingh, Bangladesh.

33. Miller, P., \& Koch, G. (2013). Newcastle disease, p 89-138. Diseases of poultry, 13th ed. WileyBlackwell, Hoboken, NJ.

34. Miller, P. J., Decanini, E. L., \& Afonso, C. L. (2010). Newcastle disease: evolution of genotypes and the related diagnostic challenges. Infection, Genetics and Evolution, 10(1), 26-35.

35. Miller, P. J., \& Koch, G. (2013). Newcastle disease. Diseases of poultry, 13, 89-138.

36. Mishra, U., \& Spradbrow, P. (1991). Present status of poultry in Nepal. Paper presented at the Newcastle Disease in Village Chickens, Control with Thermostable Oral Vaccines, Proceedings of an international workshop of ACIAR, Kuala Lumpur, Malaysia.

37. Munir, M., Abbas, M., Khan, M. T., Zohari, S., \& Berg, M. (2012). Genomic and biological characterization of a velogenic Newcastle disease virus isolated from a healthy backyard poultry flock in 2010. Virology journal, 9(1), 46. 
38. Munir, M., Cortey, M., Abbas, M., Afzal, F., Shabbir, M. Z., Khan, M. T., . . Ståhl, K. (2012). Biological characterization and phylogenetic analysis of a novel genetic group of Newcastle disease virus isolated from outbreaks in commercial poultry and from backyard poultry flocks in Pakistan. Infection, Genetics and Evolution, 12(5), 1010-1019.

39. Munir, M., Shabbir, M. Z., Yaqub, T., Shabbir, M. A., Mukhtar, N., Khan, M. R., \& Berg, M. (2012). Complete genome sequence of a velogenic neurotropic avian paramyxovirus 1 isolated from peacocks (Pavo cristatus) in a wildlife park in Pakistan. Journal of virology, 86(23), 13113-13114.

40. Munir, M., Zohari, S., Abbas, M., \& Berg, M. (2012). Sequencing and analysis of the complete genome of Newcastle disease virus isolated from a commercial poultry farm in 2010. Archives of virology, 157(4), 765-768.

41. Musiime, J. (1991). The poultry industry in Kenya with particular reference to the Newcastle disease problem. Paper presented at the Newcastle Disease in Village Chickens, Control with Thermostable Oral Vaccines, Proceedings of an international workshop of ACIAR, Kuala Lumpur, Malaysia.

42. Mustafa, M. Y., \& Ali, S. S. (2005). Prevalence of infectious diseases in local and fayoumi breeds of rural poultry (Gallus domesticus). Punjab Univ. J. Zool, 20(2), 177-180.

43. Nagai, Y., \& Klenk, H.-D. (1977). Activation of precursors to both glycoproteins of Newcastle disease virus by proteolytic cleavage. Virology, 77(1), 125-134.

44. Nagai, Y., Klenk, H.-D., \& Rott, R. (1976). Proteolytic cleavage of the viral glycoproteins and its significance for the virulence of Newcastle disease virus. Virology, 72(2), 494-508.

45. NAZIR, H. K. (2013). Issues in Poultry Sector \& Initiatives of Sindh Poultry Vaccine Centre. Presented at LDFA Expo Karachi.

46. NDV in peacock.

47. Newcastle disease hits Pakistan poultry http://www.thepoultrysite.com/poultrynews/25595/newcastle-disease-hits-pakistan-poultry.

48. Newcastle Disease Linked To Peacock Deaths In Pakistan.

49. Nguyen, T. D. (1991). Poultry production and Newcastle disease in Vietnam. Newcastle Disease in Village Chickens, Control with Thermostable Oral Vaccines, 169-170.

50. Okwor, E., \& Eze, D. (2010). Reared in South Eastern Savannah Zone of Nigeria. Research Journal of Poultry Sciences, 3(2), 23-26.

51. Rehman, H., Fawad, N., Abbas, G., Naheed, G., Siddique, B., Afzal, F., Munawar, J., Aurangzeb, M., Atif, M., Haq, I. (2013). Surveillance of poultry diseases in Punjab Province, Pakistan.

52. Rehmani, S. F., Wajid, A., Bibi, T., Nazir, B., Mukhtar, N., Hussain, A., .. Afonso, C. L. (2015). Presence of Virulent Newcastle Disease Virus in Vaccinated Chickens Farms In Pakistan. Journal of clinical microbiology, JCM. 02818-02814.

53. Sadiq, M. (2004). Pakistan poultry sector still on an upward swing. World Poult, 20, 10-11.

54. Seal, B. S., King, D. J., \& Sellers, H. S. (2000). The avian response to Newcastle disease virus. Developmental \& Comparative Immunology, 24(2-3), 257-268.

55. Shabbir, M. Z., Abbas, M., Yaqub, T., Mukhtar, N., Subhani, A., Habib, H., ... Munir, M. (2013). Genetic analysis of Newcastle disease virus from Punjab, Pakistan. Virus genes, 46(2), 309-315.

56. Shabbir, M. Z., Goraya, M. U., Abbas, M., Yaqub, T., Shabbir, M. A. B., Ahmad, A., ... Munir, M. (2012). Complete genome sequencing of a velogenic viscerotropic avian paramyxovirus 1 isolated from pheasants (Pucrasia macrolopha) in Lahore, Pakistan. Journal of virology, 86(24), 13828-13829.

57. Shabbir, M. Z., Zohari, S., Yaqub, T., Nazir, J., Shabbir, M. A. B., Mukhtar, N., . . Abbas, M. (2013). Genetic diversity of Newcastle disease virus in Pakistan: a countrywide perspective. Virology journal, 10(1), 170. 
58. Sharif, A., Ahmad, T., Umer, M., Rehman, A., \& Hussain, Z. (2014). Prevention and control of Newcastle disease. International Journal of Agriculture Innovations and Research, 3(2), 454-460.

59. Siddique, M., Sabri, M., \& Khan, M. (1986). Outbreak of Newcastle disease in vaccinated chicken flocks in and around Faisalabad [Pakistan]. Pakistan Veterinary Journal (Pakistan).

60. Strausbaugh, L. J., \& Berkelman, R. L. (2003). Human illness associated with use of veterinary vaccines. Clinical infectious diseases, 37(3), 407-414.

61. Tan, L.-T., Xu, H.-Y., Wang, Y.-L., Qin, Z.-M., Sun, L., Liu, W.-J., \& Cui, Z.-Z. (2008). Molecular characterization of three new virulent Newcastle disease virus variants isolated in China. Journal of clinical microbiology, 46(2), 750-753.

62. Tauqir, N., \& Nawaz, H. (2001). Performance and economics of broiler chicks fed on rations supplemented with different levels of sodium bentonite. Int. J. Agric. Biol, 3, 149-150.

63. Toyoda, T., Sakaguchi, T., Imai, K., Inocencio, N. M., Gotoh, B., Hamaguchi, M., \& Nagai, Y. (1987). Structural comparison of the cleavage-activation site of the fusion glycoprotein between virulent and avirulent strains of Newcastle disease virus. Virology, 158(1), 242-247.

64. Wajid, A., Dimitrov, K. M., Wasim, M., Rehmani, S. F., Basharat, A., Bibi, T., . . Ababneh, M. (2017). Repeated isolation of virulent Newcastle disease viruses in poultry and captive nonpoultry avian species in Pakistan from 2011 to 2016. Preventive Veterinary Medicine, 142, 1-6.

65. Wajid, A., Dundon, W. G., Hussain, T., \& Babar, M. E. (2018). Pathotyping and genetic characterization of avian avulavirus-1 from domestic and wild waterfowl, geese and black swans in Pakistan, 2014 to 2017. Archives of virology, 1-6. 